\title{
Calculation of Electromagnetic Fields in Electrical Machines using Finite Elements Method
}

\author{
Vasilija Sarac, Goce Stefanov \\ University Goce Delcev, Electrotechnical Faculty, P.O. Box 201, 2000 Stip, Macedonia \\ vasilija.sarac@ugd.edu.mk,goce.stefanov@ugd.edu.mk \\ doi:10.4156/ ijei.vol2. issue1.3
}

\begin{abstract}
Finite Elements Method is a contemporary numerical method for determination of distribution of electromagnetic fields inside different objects. In this paper three different induction motors will be analyzed : Solid Salient Poles Synchronous Motor, product of company MAWDSLEY, Single Phase Shaded Pole Motor, product of company Micron-Tech and Induction Squirrel Cage Motor, product of company Koncar. In all analyzed motors distribution of electromagnetic field and magnetic flux density are calculated using two different approaches: magneto-static at zero herz frequency and magneto-dynamic at fifty herz frequency. Further on, calculation of different motor parameters such as magnetic flux density distribution in motor air gap at different operating regimes is performed and adequate conclusion are derived.
\end{abstract}

Keywords: Finite Elements Method, Magnetic Fields Calculation, Induction Motors

\section{Introduction}

Finite Elements Method (FEM) is a proven numerical tool for analysis of electromagnetic phenomena in electrical machines and devices. This method enables to enter "inside the machine” and to evaluate exactly the magnetic quantities such as air gap flux density in any part of the machine. On the basis of analyses of spatial distribution of flux density in each part of the machine, week point in magnetic core can be discovered. On that way, FEM has proven itself as a valuable tool in motor design. In this paper a non-linear magnetic field analysis is performed using two different approaches: magneto-static where all electromagnetic quantities are analyzed in exact moment of time, meaning frequency $\mathrm{f}=0 \mathrm{~Hz}$ and time harmonic magnetic approach where the magnetic field inside the machine is time varying, meaning frequency $\mathrm{f}=50 \mathrm{~Hz}$ [1]. Three different induction motors are analyzed: Solid Salient Synchronous Motor-SSPSM, product of MAWDSLEY, Single Phase Shaded Pole MotorSPSPM, product of Micron-Tech and Induction Squirrel Cage Motor -ISQM, product of Koncar (Fig.1) . In order to enable FEM calculation to be applicable on different motor models, for each motor model, exact motor geometry and material characteristics in different motor regions is input in FEM pre-processing part. Very important issue is to define boundary conditions on outer motor geometry and in this case Dirichlet-boundary conditions are used. Another important subject in motor modeling is definition of the mesh of finite elements. By dividing the motor's cross section into large number of regions i.e. elements with simple geometry (triangles) the true solution of magnetic vector potential is approximated by a very simple function.

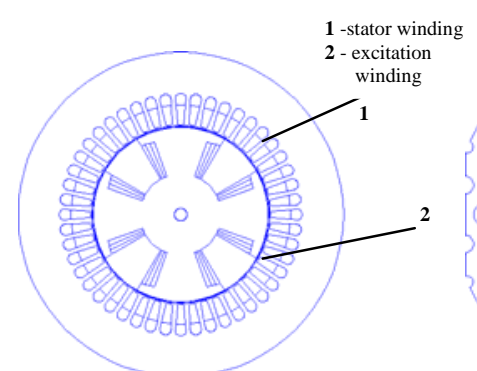

(a) SSSM

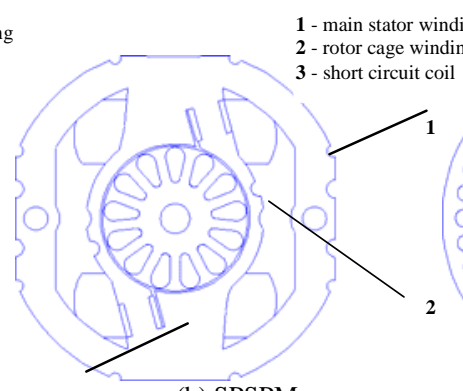

3 (b) SPSPM

Figure 1. Cross-section of different motors models in FEM

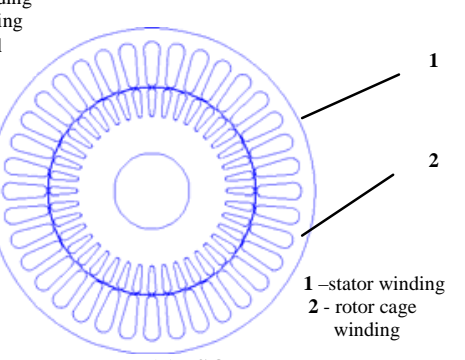

(c) ISQM 


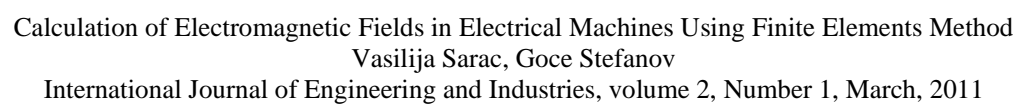

In time-harmonic motor models only stator current is input and consequently currents in other motor windings are freely induced. On that way analysis of electromagnetic phenomena inside the motors is closer to the real electromagnetic process inside the machines when they are supplied with voltage 220 $\mathrm{V}, 50 \mathrm{~Hz}$ or $380 \mathrm{~V}, 50 \mathrm{~Hz}$. Both analyses are performed in 2D domain.

\section{Modeling of motor models for FEM}

FEM analysis of the motor is divided into three parts: pre-processing, processing and postprocessing part. In pre-processing part motor geometry is defined as well as material properties. Calculation of currents is performed, boundary conditions on outer motor geometry and finite element mesh are also defined. In order processing part to be executed and output results to be obtained system of Maxwell's equation should be solved. They differ in both cases: magnetostatic and time-harmonic.

Evaluation of exact magnet quantities inside the motor, is subject to numerical calculation of magnetic vector potential and flux density. FEM has possibility to solve magnetic vector potential and consequently magnetic flux density by solving relevant set of Maxwell equations for magneto-static case as well as for magneto-dynamic i.e. time harmonic case. Magnetostatic problems are problems in which the fields are time-invariant. In this case field density $\mathbf{H}$ and flux density B must obey:

$$
\begin{aligned}
& \nabla x \mathbf{H}=\mathbf{J} \\
& \nabla x \mathbf{B}=0
\end{aligned}
$$

subject to a constitute relation between $\mathrm{B}$ and $\mathrm{H}$ for each material:

$$
B=\mu H
$$

FEM goes about finding a field that satisfies (1)-(3) via a magnetic vector potential. Flux density is written in terms of vector potential A as:

$$
B=\nabla x A
$$

For the magneto-static problem and non-linear B-H relation FEM solves the equitation:

$$
\nabla x\left(\frac{1}{\mu(B)} \nabla x A\right)=J
$$

The advantage of using the vector-potential formulation is that all the conditions to be satisfied have been combined into a single equation. If $\mathrm{A}$ is found, $\mathrm{B}$ and $\mathrm{H}$ can be deduced by differentiating A.

After exact motor geometry is input boundary conditions are defined. For these specific motor models are chosen Dirichlet boundary conditions e.g. $A=0$. The most common use of Dirichlet-type boundary conditions in magnetic problems is to define $\mathrm{A}=0$ along a boundary to keep the magnetic flux from crossing the boundary. After the boundary conditions are defined, properties of all materials are input in motor models. Besides inputting the magnetization curve as $\mathrm{B}=\mathrm{f}(\mathrm{H})$ also the lamination of magnet material is input according to Fig. 2 (a) as well as fill factor. The result of this model is that one can account for laminations with hysteresis and eddy currents. 


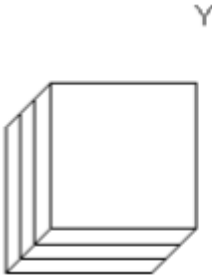

(a)

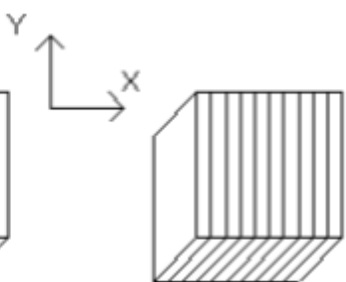

(b)

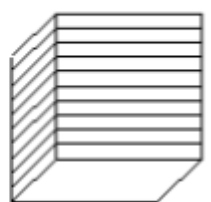

(c)

Figure 2. Possibilities for different motor laminations

In order magnetic vector potential A to be determined it is necessary the whole domain i.e. motor's cross-section to be divided into numerous elements. In Fig.3 is presented finite element mesh for each of the motor models.

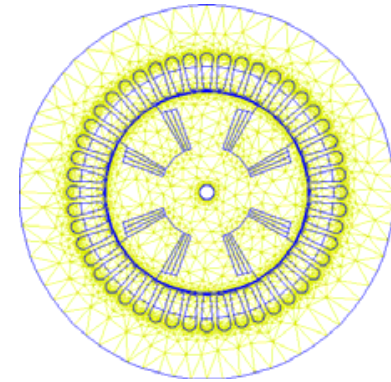

(a) SSPSM

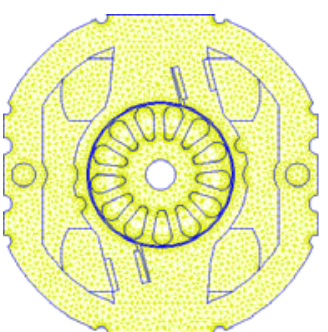

(b) SPSPM

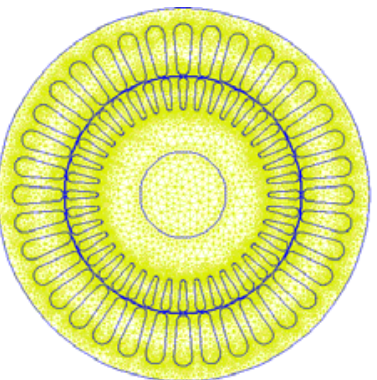

(c) ISQM

Figure 3. Finite Elements mesh in motor models cross-section

Inputting of current densities in all motor models is done on consequently manner. For SPSPM first in main stator winding current density is input, than from the value of magnetic flux in short circuit coil current in the coil is calculated and input in motor model. Finally having the both stator currents and from the value of magnetic flux in motor air gap, current in rotor squirrel cage winding is calculated. Having all three currents in motor model the program is run at stator frequency $\mathrm{f}=0 \mathrm{~Hz}$. Similar philosophy is followed in inputting the current densities in SSPSM and ISQM.

When analysing induction machines, considering their AC excitation the air gap magnetic field is always a time-varying quantity . In materials with non-zero conductivity eddy currents are induced, consequently the field problem turns into magneto-dynamic i.e. non-linear time harmonic problem. When rotor is moving, the rotor quantities oscillate at slip frequency . In this case the rotor bars conductivity $\sigma$ is adjusted corresponding to the slip. Consequently following partial equation is going to be solved numerically:

$$
\nabla x\left(\frac{1}{\mu(B)} \nabla x A\right)=-\sigma \dot{A}+J_{s r c}-\sigma \nabla V
$$

where $J_{\text {src }}$ represents the applied current sources. The additional voltage gradient $\nabla V$ in 2$\mathrm{D}$ field problems is constant over the conduction body.

Strictly speaking the permeability $\mu$ should be constant for harmonic problems. However, FEM retains a nonlinear relationship in the harmonic formulation, allowing the program to approximate the effects of saturation on the phase and amplitude of the field distribution. FEM also allows for the inclusion of complex and frequency-dependant permeability in time harmonics. These features allow the program to model materials with thin laminations and approximately model hysteresis effects.

Program is run at constant frequency $\mathrm{f}=50 \mathrm{~Hz}$. In SPSPM model is input only current in main stator winding while currents in short circuit coil and rotor winding are freely induced. In 
SSPSM currents are input in both motor windings: excitation and main stator winding while in ISQM currents are input only in stator winding and in rotor cage winding they are freely induced.

\section{Post-processing results}

After preparing all motor models programs are run and post-processing results such as magnetic field distribution in motor's cross section as well as spatial distribution of magnetic flux density in the middle air gap line are obtained. All results are presented for magnetostatic and time-harmonic case.

\subsection{Results from magneto-static analysis}

In Fig. 4 a) and b) is presented respectively magnetic flux distribution at SSPSM when only the stator winding is energized and when only the excitation winding is energized while in Fig. 5 a) and b) is presented magnetic flux distribution when both winding are energized simultaneously with rated currents as well as spatial distribution of magnetic flux density in the middle of the air gap consequently.

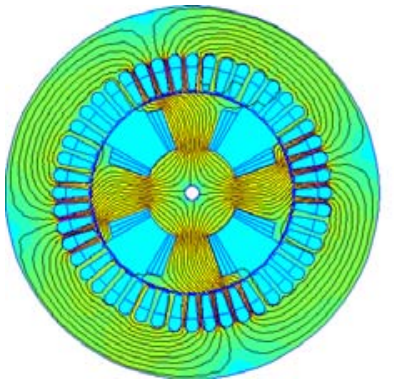

(a)
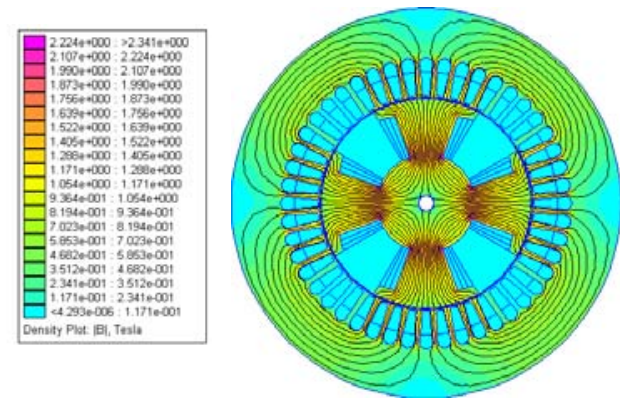

(b)

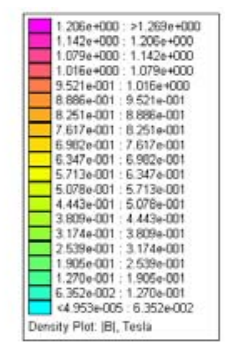

Figure 4. Magnetic flux distribution at SSPSM, stator and excitation winding separately energized

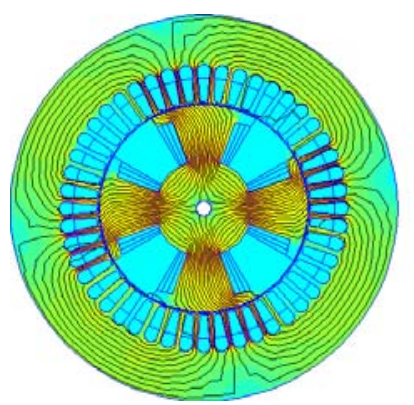

(a)

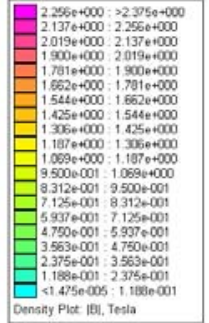

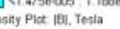

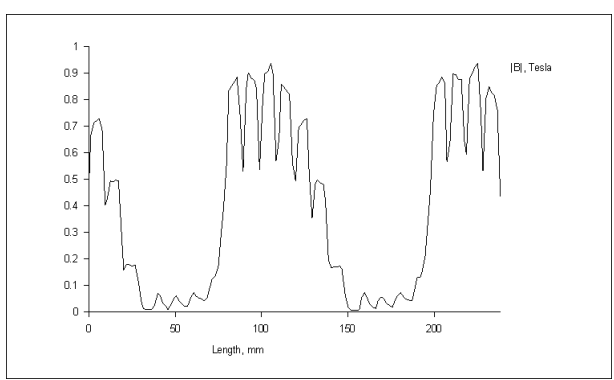

(b)

Figure 5. Flux distribution at SSPSM, in motor cross-section and air gap, both windings energized

Calculation of electromagnetic fields inside electrical machines is extended by applying FEM method on SPSPM. In spite of its simple construction SPSPM is very complex due to the existence of three magnetically coupled windings which produce an elliptic rotating magnetic filed in motor's air gap. Therefore, quite extended procedure of input of all three currents in all three windings, is implemented. Current density in short circuit coil is calculated from induced voltage in the coil due to the flux in the short circuit coil produced by main stator winding, while current density in rotor bars is calculated from induced voltage due to the existence of air gap flux from main stator winding and short circuit coil. In Figure 6 a), b) and c) is presented magnetic flux distribution when only main stator winding, short circuit coil and rotor winding 
are separately excited with no-load currents. In Figure 7 a) is presented magnetic flux distribution in motor cross section at no-load operating condition when all three windings are energized with no-load currents. Spatial distribution of magnetic flux density in the motor air gap at no-load is presented in Figure 7 b). Results of flux distribution in motor cross section and in the air gap, from all three winding and for rated load operation, are presented in Figure 8 a) and b) respectively. Locked rotor operating regime of SPSPM is presented in Figure 9. Different motor operating regimes are simulated by taking into consideration rotor slip and consequently the impedance of all rotor bars is adjusted correspondingly to the value of rotor slip. Separate methodology is implemented for modeling rotor skew by dividing motor axial length into n-pieces and each piece is rotated with respect to the neighbor one for an angle $\alpha / n$, where $\alpha$ is the angle of rotor skewing. In analyzed SPSPM, angle of rotor skewing is $17^{\circ}$ and motor axial length is $\mathrm{l}_{\delta}=16 \mathrm{~mm}$.

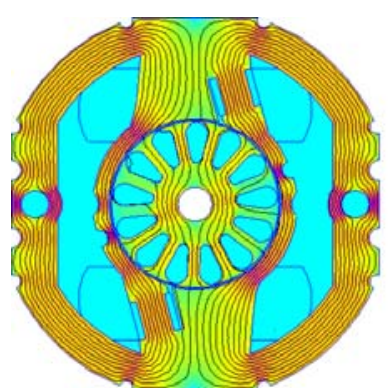

(a)
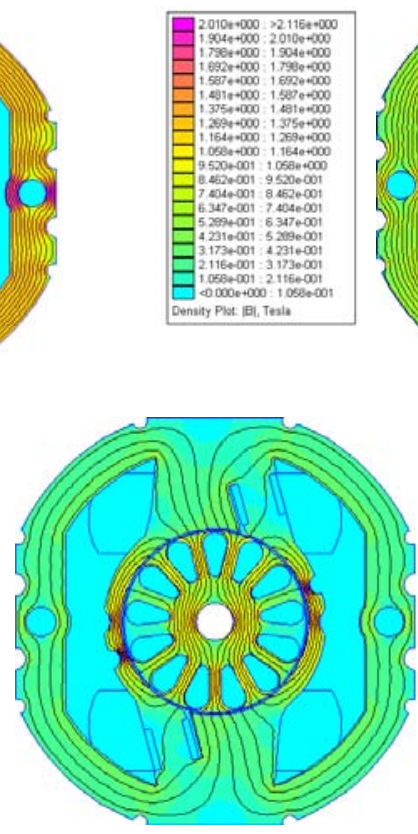

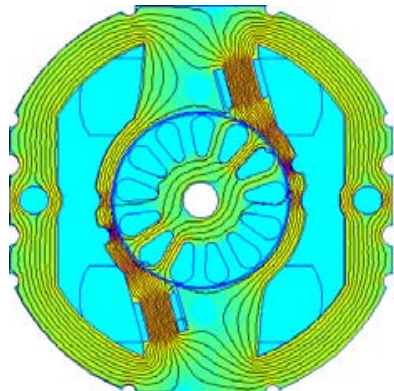

(b)
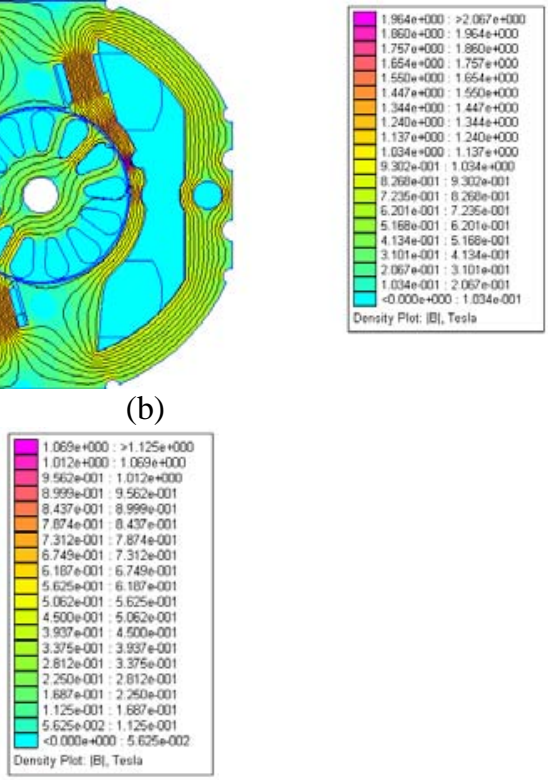

(c)

Figure 6. Flux distribution at SPSPM all three winding separately energized at no-load

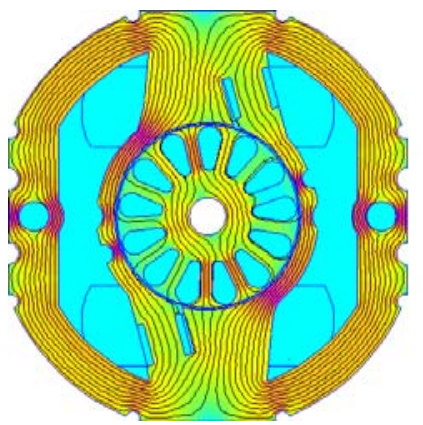

(a)

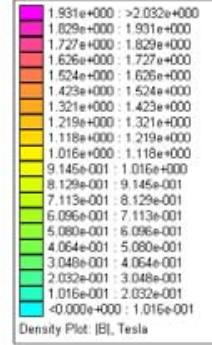

Figure 7

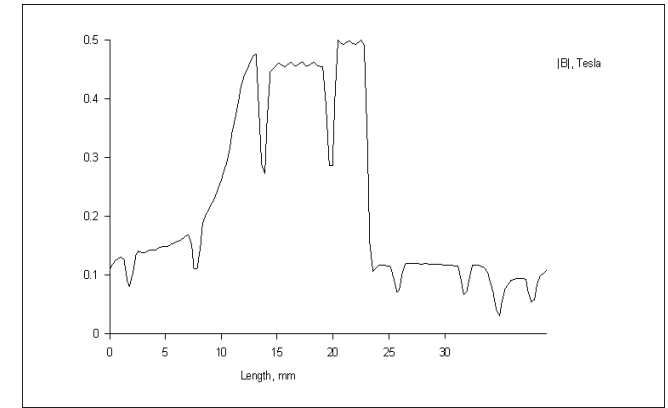

(b) 


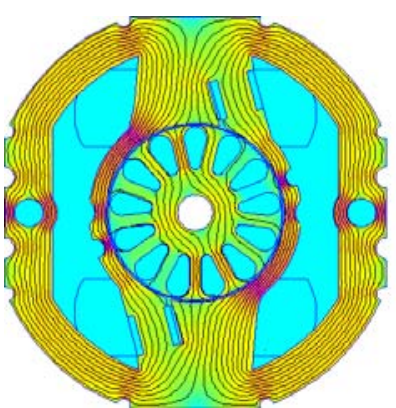

(a)
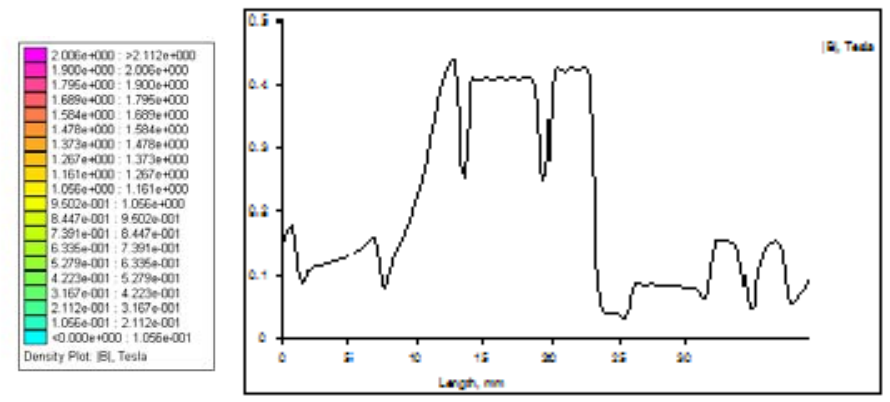

(b)

Figure 8. Flux distribution at SPSPM all windings energized at rated load

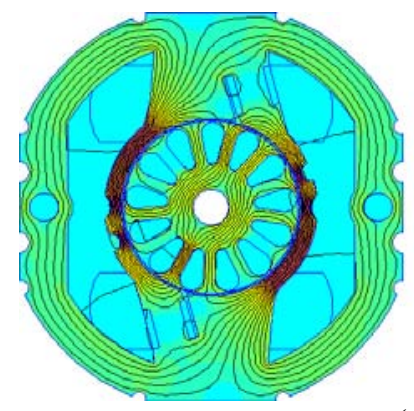

(a)
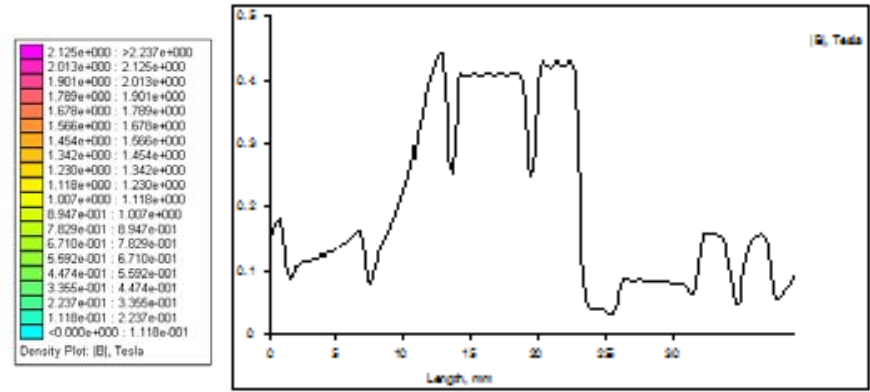

(b)

Figure 9. Flux distribution at SPSPM all windings energized at locked rotor

Induction squirrel cage motor is a third object of investigation where FEM method for calculation of electromagnetic fields is applied. Current densities are input in both motor windings by taking into consideration that motor model is magneto-static, meaning that all magnetic phenomena inside the motor are analyzed in certain moment of time, so in this analysis it is adopted that this moment is when current in phase A has maximal value and currents in other two phases are half of the value of the current in phase A. Currents phase displacement is solved by adopting the adequate sign of the currents in each phase and by taking into consideration the fact that analyzed motor has four poles. Different motor operating regimes are analyzed and in Figure 10 a) and b) is presented magnetic filed distribution in motor cross section as well as in air gap for no load operating condition when all motor windings are energized with no-load currents while in Figures 11 and 12 is presented magnetic flux distribution at rated load and locked-rotor operating condition.

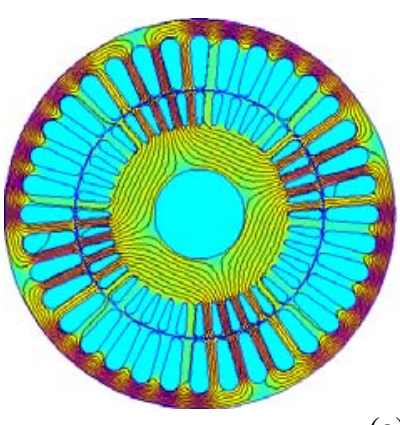

(a)

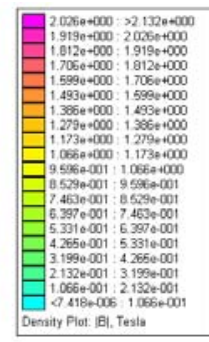

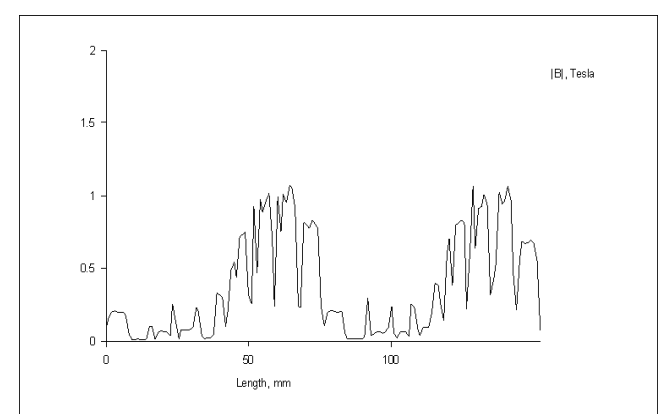

(b)

Figure 10. Flux distribution at ISQM all windings energized at no-load 


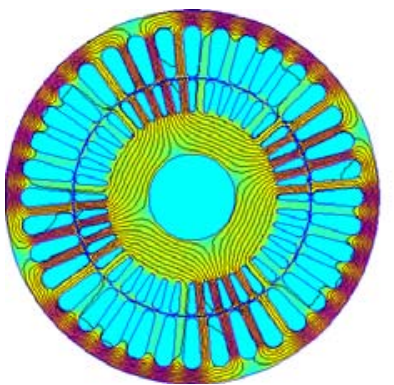

(a)

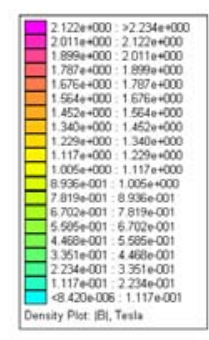

Figure 1

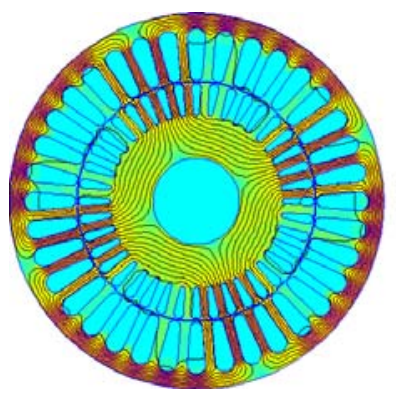

(a)

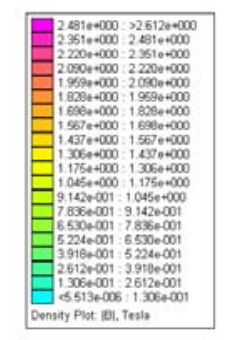

Figure 12. Flux distribution at ISQM

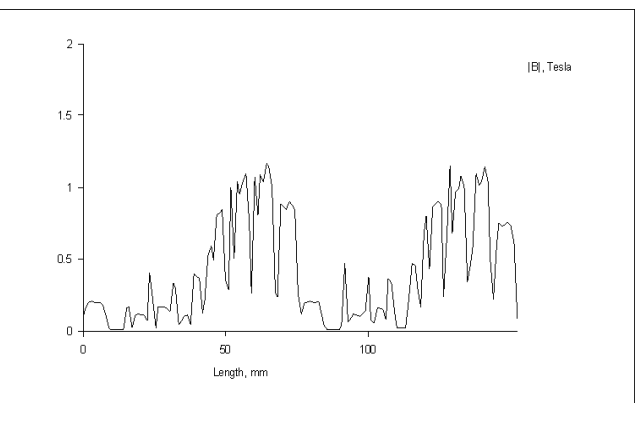

(b)

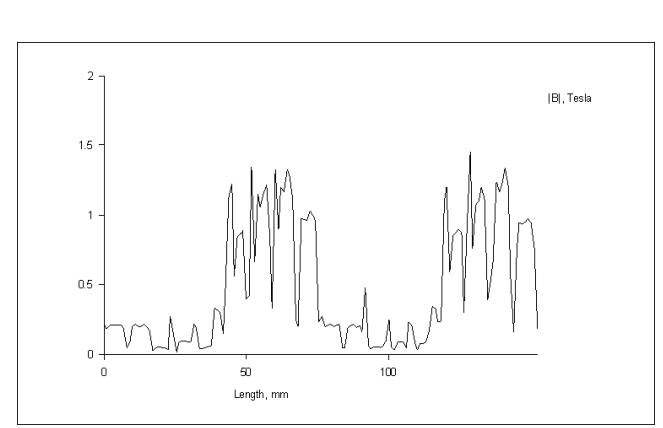

(b)

\subsection{Results from time-harmonic analysis}

Time-harmonic analysis at $\mathrm{f}=50 \mathrm{~Hz}$ and for different operating regimes, is run on SSPSM, SPSPM and ISQM. This analysis is much closer to the real processes which occur inside the machines, since in SPSPM only stator and short circuit coil are excited while in rotor winding currents are freely induced due to the existence of rotating electromagnetic field inside motor air gap [2]. At ISQM only stator winding is energized and in squirrel cage rotor winding currents are freely induced. Both motor windings, stator and excitation rotor winding, are energized in SSPSM since both windings contribute towards rotation of the motor with synchronous speed. Further on, in asynchronous motors, slip is also taken into consideration by adjusting the rotor bars conductivity correspondingly to the slip, i.e. motor operating regime. In Figure 13 a) b) and c) is presented magnetic flux distribution in cross-sections of SSPSM, SPSPM and ISQM respectively for no-load operating condition. 


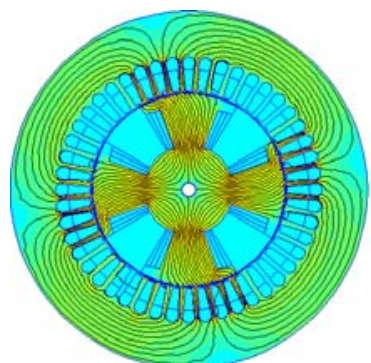

(a)

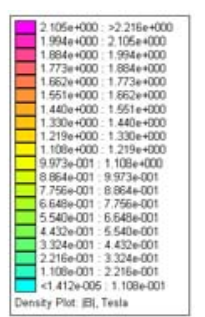

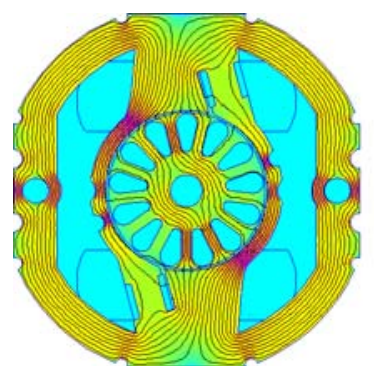

(b)
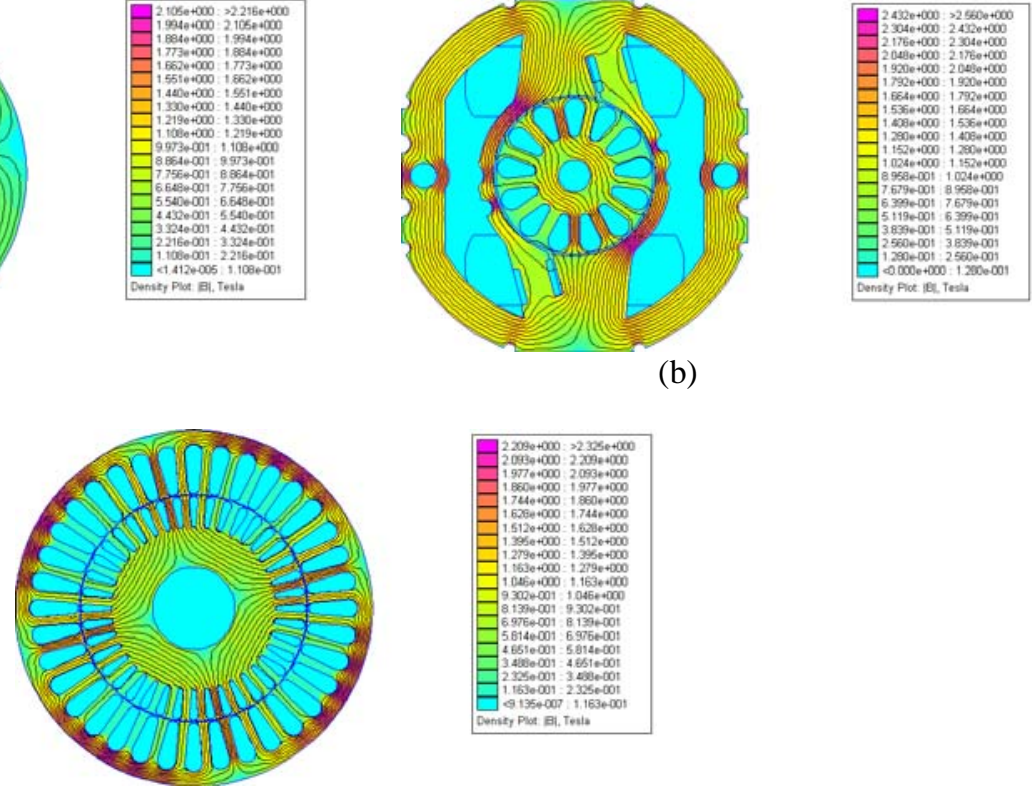

(c)

Figure 13. Flux distribution in motors cross-section in time-harmonic domain at no-load

In Fig. 14 a), b) and c) is presented magnetic flux distribution at rated load condition for SSPSM, SPSPM and ISQM consequently. As it can be concluded from comparison of Figs. 13 and 14 values of magnetic flux density are higher at rated operation condition due to the higher vales of stator and rotor currents. Obtained vales of flux density at rated load conditions in time-harmonic domain for SSPSM and SPSPM are rather high in some critical points of motor construction i.e. stator bridge of SPSPM and permanent magnet rotor for SSPSM which opens the possibility for further research by implementation of soft magnetic materials in motor construction .

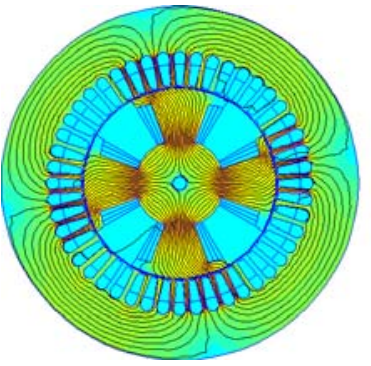

(a)
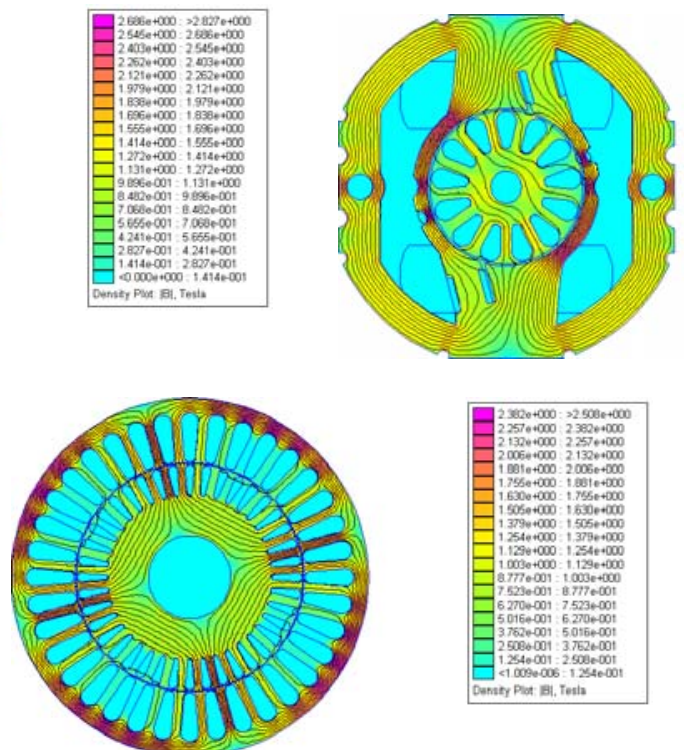

(c)

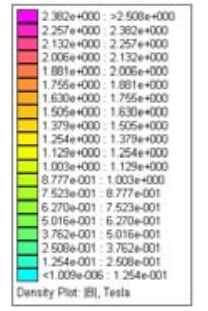

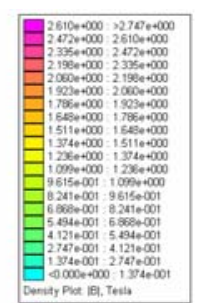

(b)

Figure 14. Flux distribution in motors cross-section in time-harmonic domain at rated load 


\section{Conclusion}

Finite Elements Method is applied in three different motor models (solid salient synchronous motor, single phase shaded pole motor and induction squirrel cage motor) in order to be obtained magnetic flux distribution inside the motor models. Two different approaches are implemented in magnetic filed calculation: magnetostatic, at $\mathrm{f}=0 \mathrm{~Hz}$ and magneto-dynamic at $\mathrm{f}=50 \mathrm{~Hz}$. Magnetostatic approach involves consequent calculation of current densities in each motor winding as a result of the flux from the excitation winding. In magneto-dynamic i.e. time-harmonic approach, currents are freely induced in rotor windings due to the rotating electromagnetic filed in machine air-gap. On that way calculation of electromagnetic process inside the machines is much closer to the real ones. In magneto-dynamic approach rotor slip is also taken into consideration by adjusting rotor windings conductivity correspondingly to the slip. Skewing of rotor bars is also taken into consideration by discretization of motor axial dimensions into n-pieces and their adequate rotation. Obtained values of magnetic flux density and its distribution in all motor models, by these two approaches show satisfactory agreement which proves the accuracy of motor models. In time-harmonic domain values of magnetic flux density at rated load are higher than at no-load. This is comprehensible considering the higher values of currents in all motor windings at rated load. Higher values of magnetic flux density in time-harmonic domain in some critical parts of motor construction can be further reduced by implementing soft magnetic powders in motor design.

\section{References}

[1] V.Sarac, G. Cvetkovski, “Different Motor Models Based on Parameter Variation using Method of Genetic Algorithms”, Journal Prezglad Elektrotechniczny (Electrical Review), ISSN 0033-2097, R 87 NR 3/2011., pp. 162-165.

[2] V.Sarac, "Different Approaches in Numerical Analysis of Electromagnetic Phenomena in Shaded Pole Motor with Application of Finite Elements Method,”, In Proceeding(s) of the EMTS International Symposium of Electromagnetic Theory , pp.97-100, 2010.

[3] V.Sarac, G.Stefanov, G. Cvetkovski, "Influence of Number of Varied Parameters on Torque of Single Phase Optimized Motor Models”, IGTE International Symposium of Numerical Field Calculation in Electrical Engineering, Book of Abstracts, pp.45-46, 2010.

[4] D. Meeker, Finite Element Magnetics, Users Manual for FEMM Ver. 4.2, Boston, Massachusets, USA, 2009. 